\title{
THE GENEVA CONVENTIONS OF AUguST, 12, $1949^{1}$
}

\section{Some publications}

Sw. fr

The Geneva Conventions of August 12, 1949. 2nd Ed. 1950. $245 \mathrm{pp}$.

Commentary published under the General Editorship of Mr. J. Pictet, member of ICRC and Director-General:

- Vol. 1: Geneva Convention for the Amelioration of the Condition of the Wounded and Sick in Armed Forces in the Field - $466 \mathrm{pp}$.

$\begin{array}{rr}\text { bound } & 18 .- \\ \text { paper-back } & 15 .-\end{array}$

- Vol. 2: Geneva Convention for the Amelioration of the Condition of Wounded, Sick and Shipwrecked Members of Armed Forces at Sea $320 \mathrm{pp}$.

$\begin{array}{rr}\text { bound } & 23 .- \\ \text { paper-back } & 18 .-\end{array}$

- Vol. 3: Geneva Convention relative to the Treatment of Prisoners of War $-764 \mathrm{pp}$.

$\begin{aligned} \text { bound } & 38 .- \\ \text { paper-back } & 33 .-\end{aligned}$

- Vol. 4 : Geneva Convention relative to the Protection of Civilian Persons in Time of War $-660 \mathrm{pp}$.

$\begin{array}{rr}\text { bound } & 33 .- \\ \text { paper-back } & 28 .-\end{array}$

The Geneva Conventions of August, 12, 1949:

- Brief Summary for Members of Armed Forces and the General Public, 13 pp. . . . . . . . . . . . . . . . . . . . . 1.50

- Course of Five Lessons, 102 pp. . . . . . . . . . . . . . . . . . . 7.-

- Essential Provisions, 4 pp. . . . . . . . . . . . . . . . . . 0.30

\section{Transparencies}

The Geneva Conventions of August, 12, 1949:

- Thirty Slides with Comments, 33 pp. mimeographed . . . . . . . . . 25.-

1) These publications and slides may be obtained from the ICRC Press and Information Service, 7, avenue de la Paix, $\mathrm{CH}-1211$ Geneva 1. 


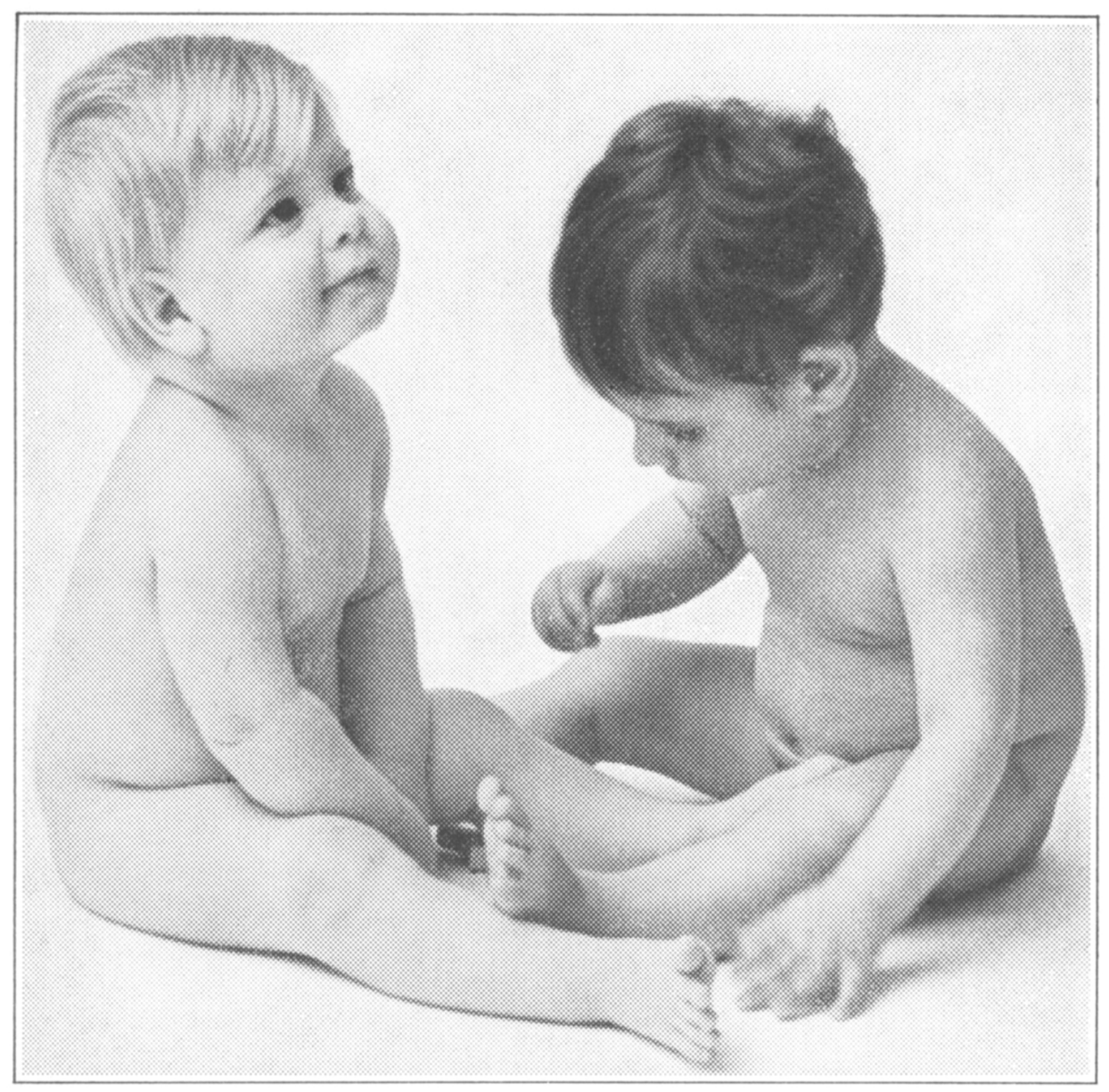

Nestlé devoted to childcare throughout the world

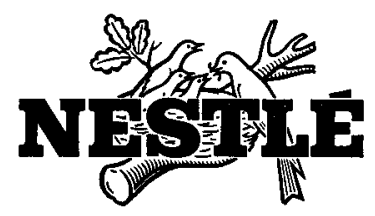



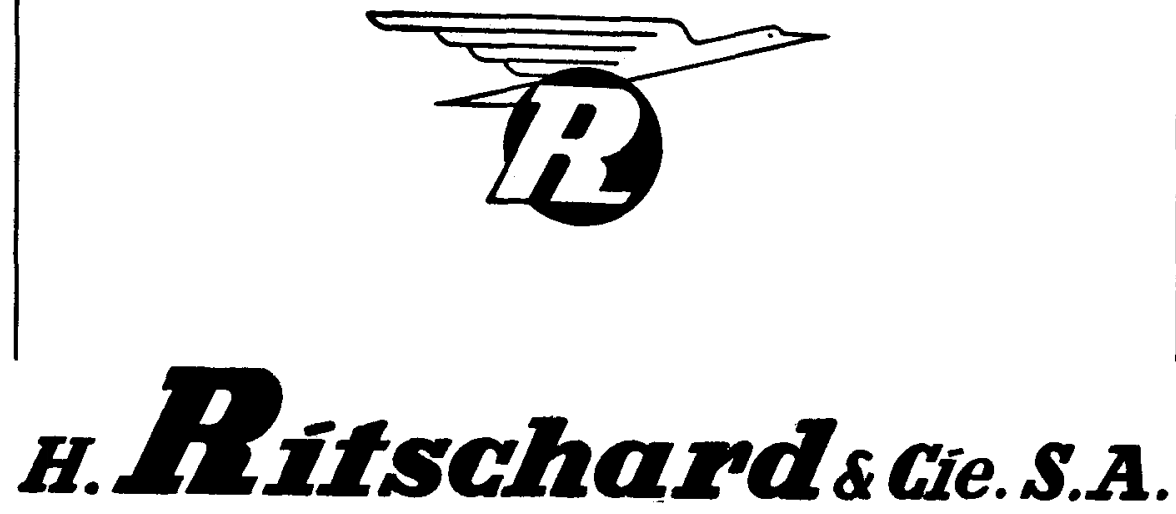

INTERNATIONAL TRANSPORT TRAVEL AGENCY

\section{GENEVA, 49, route des Jeunes}

Telephone 437600 - Teleprinter 22167

Travel agency: Branch office in Geneva:

18 , place Cornavin - $\varnothing 323402$

\section{Exchange - Tickets - Sea passages \\ Insurance - Customs Agency \\ Road haulage - Storage}

Home delivery of air and rail tickets on request by telephone

Branches :

LAUSANNE - ANNEMASSE (France) 


\section{ADDRESSES OF CENTRAL COMMITTEES}

AFGHANISTAN - Afghan Red Crescent, Kabul. ALBANIA - Albanian Red Cross, 35, Rruga Barrikadavet, Tirana.

ALGERIA - Central Committee of the Algerian Red Crescent Society, 15 bis, Boulevard Mohamed V, Algiers.

ARGENTINE - Argentine Red Cross, H. Yrigoyen 2068, Buenos Aires.

AUSTRAIIA - Australian Red Cross, 122-128 Flinders Street, Melbourne, C. 1.

AUSTRIA - Austrian Red Cross, 3 Gusshausstrasse, Postfach 39, Vienna IV.

BELGIUM - Belgian Red Cross, 98, Chaussée de Vleurgat, Brussels 5.

BOLIVIA - Bolivian Red Cross, Avenida Simon Bolivar, 1515 (Casilla 741), La Paz.

BRAZIL - Brazilian Red Cross, Praça da Cruz Vermelha $12 \mathrm{c} 2 / 86$, Rio de Janeiro.

BULGARIA - Bulgarian Red Cross, 1, Boul. S.S. Biruzov, Sofia.

BURMA - Burma Red Cross, 42, Strand Road, Red Cross Building, Rangoon.

BURUNDI - Red Cross Society of Burundi, rue du Marché 3, P.O. Box 1324, Bujumbura.

CAMBODIA - Cambodian Red Cross, 17, Vithei Croix-Rouge, P.O.B. 94, Phnom-Penh.

CAMEROON - Central Committee of the Cameroon Red Cross Society, rue HenryDunant, P.O.B. 631, Yaoundé.

CANADA - Canadian Red Cross, 95 Wellesley Street, East, Toronto 284 (Ontario).

CEYLON - Ceylon Red Cross, 106 Dharmapala Mawatte, Colombo VII.

CHILE - Chilean Red Cross, Avenida Santa Maria 0150, Casilla 246 V., Santiago de Chile.

CHINA - Red Cross Society of China, 22 Kanmien Hutung, Peking, E.

COLOMBIA - Colombian Red Cross, Carrera 7a, 34-65 Apartado nacional 1110, Bogotá D.E.

CONGO - Red Cross of the Congo, 41, Avenue Valcke P.O. Box 1712, Kinshasa.

COSTA RICA - Costa Rican Red Cross, Calle 5a, Apartado 1025, San José.

CUBA - Cuban Red Cross, Calle 23201 esq. N. Vedado, Havana.

CZECHOSLOVAKIA - Czechoslovak Red Cross, Thunovska 18, Prague $I$.

DAHOMEY - Red Cross Society of Dahomey, P.O. Box 1, Porto Novo.

DENMARK - Danish Red Cross, Ny Vestergade 17, Copenhagen $K$.

DOMINICAN REPUBLIC - Dominican Red Cross, Calle Galvan 24, Apartado 1293, Santo Domingo.

ECUADOR - Ecuadorean Red Cross, Calle de la Cruz Roja y Avenida Colombia 118, Quito.

ETHIOPIA - Ethiopian Red Cross, Red Cross Road No. 1, P.O. Box 195, Addis Ababa.
FINI.AND - Finnish Red Cross, Tehtaankatu $1 \mathrm{~A}$, Box 14168, Helsinki 14.

FRANCE - French Red Cross, 17, rue Quentin Bauchart, Paris (8c).

GERMANY (Dem. Republic) - German Red Cross in the German Democratic Republic, Kaitzerstrasse 2, Dresden A. 1.

GERMANY (Federal Republic) - German Red Cross in the Federal Republic of Germany, Friedrich-Ebert-Allee 71, 5300 Bonn 1, Postfach (D.B.R.)

GHANA - Ghana Red Cross, P.O. Box 835, Accra.

GREAT BRITAIN - British Red Cross, 9 Grosvenor Crescent, London, S.W.1.

GREECE - Hellenic Red Cross, rue Lycavittou 1, Athens 135.

GUATEMALA - Guatemalan Red Cross, 3.0 Calle 8-40 zona 1 , Guatemala C.A.

GUYANA - Guyana Red Cross, P.O. Box 351, Eve Leary, Georgetown.

HAITI - Haiti Red Cross, Place des Nations Unies, B.P. 1337, Port-au-Prince.

HONDURAS - Honduran Red Cross, Calle Henry Dunant 516, Tegucigalpa.

HUNGARY - Hungarian Red Cross, Arany Janos utca 31, Budapest $V$.

ICELAND - Icelandic Red Cross, Ø]duggøtu 4, Reykjavik, Post Box 872.

INDIA - Indian Red Cross, 1 Red Cross Road, New Delhi 1 .

INDONESIA - Indonesian Red Cross, Tanah Abang Barat 66, P.O. Box 2009, Djakarta.

IRAN - Iranian Red Lion and Sun Society, Avenue Ark, Teheran.

IRAQ - Iraqi Red Crescent, Al-Mansour, Baghdad.

IRELAND - Irish Red Cross, 16 Merrion Square, Dublin 2.

ITALY - Italian Red Cross, 12, via Toscana, Rome.

IVORY COAST - Ivory Coast Red Cross Society, B.P. 1244, Abidjan.

JAMAICA - Jamaica Red Cross Society, 76 Arnold Road, Kingston 5.

JAPAN - Japanese Red Cross, 5 Shiba Park, Minato-Ku, Tokyo.

JORDAN - Jordan National Red Crescent Society, P.O. Box 10 001, Amman.

KENYA - Kenya Red Cross Society, St Johns Gate, P.O. Box 712, Nairobi.

KOREA (Democratic People's Republic) - Red Cross Society of the Democratic People's Republic of Korea, Pyongyang.

KOREA (Republic) - The Republic of Korea National Red Cross, 32-3 Ka Nam San-Donk, Seoul.

KUWAIT - Kuwait Red Crescent Society, P.O. Box 1359, Kuwait.

LAOS - Lao Red Cross, P.B. 650, Vientiane.

LEBANON — Lebanese Red Cross, rue Général Spears, Beirut. 


\section{ADDRESSES OF CENTRAL COMMITTEES}

LIBERIA - Liberian National Red Cross, National Headquarters, Corner of Tubman boulevard and 9 th Street Sinkor, P.O. Box 226, Monrovia.

LIBYAN ARAB REPUBLIC - Libyan Red Crescent, Berka Omar Mukhtar Street, P.O. Box 541, Benghazi.

LIECHTENSTEIN - Liechtenstein Red Cross, Vaduz.

LUXEMBOURG - Luxembourg Red Cross, Parc de la Ville, C.P. 234, Luxembourg.

MADAGASCAR - Red Cross Society of Madagascar, rue Clemenceau, P.O. Box 1168, Tananarive.

MALAYSIA - Malaysian Red Cross Society, 519 Jalan Belfield, Kuala Lumpur.

MALI - Mali Red Cross, B.P. 280, route de Koulikora, Bamako.

MEXICO - Mexican Red Cross, Avenida Ejército Nacional, $\mathrm{n}^{\circ} 1032$, Mexico 10, D.F.

MONACO - Red Cross of Monaco, 27 Boul. de Suisse, Monte-Carlo.

MONGOLIA - Red Cross Society of the Mongolian People's Republic, Central Post Office, Post Box 537, Ulan Bator.

MOROCCO - Moroccan Red Crescent, rue Benzakour, B.P. 189, Rabat.

NEPAL - Nepal Red Cross Society, Tripureswore, P.B. 217, Kathmandu.

NETHERLANDS - Netherlands Red Cross, 27 Prinsessegracht, The Hague.

NEW ZEALAND - New Zealand Red Cross, 61 Dixon Street, P.O.B. 6073, Wellington C.2.

NICARAGUA - Nicaraguan Red Cross, 12 Avenida Noroeste, Managua, D.N.

NIGER - Red Cross Society of Niger, B.P. 386, Niamey.

NIGERIA - Nigerian Red Cross Society, Eko Akete Close, off. St. Gregory Rd., Onikan, P.O. Box 764, Lagos.

NORWAY - Norwegian Red Cross, Parkveien 33b, Oslo.

PAKISTAN - Pakistan Red Cross, Frere Street, Karachi 4.

PANAMA - Panamanian Red Cross, Apartado 668, Panama.

PARAGUAY - Paraguayan Red Cross, calle André Barbero y Artigas 33, Asunción.

PERU - Peruvian Red Cross, Jiron Chancay 881, Lima.

PHILIPPINES — Philippine National Red Cross, 860 United Nations Avenue, P.O.B. 280, Manila.

POLAND - Polish Red Cross, Mokotowska 14, Warsare.

PORTUGAL - Portuguese Red Cross, General Secretaryship, Jardim 9 de Abril, 1 a 5, Lisbon 3.

RUMANIA - Red Cross of the Socialist Republic of Rumania, Strada Biserica Amzei 29, Bucarest.

SALVADOR - Salvador Red Cross, 3a Avenida Norte y 3a Calle Poniente 21, San Salvador.
SAN MARINO - San Marino Red Cross, Palais gouvernemental, San Marino.

SAUDI ARABIA - Saudi Arabian Red Crescent Riyadh.

SENEGAL - Senegalese Red Cross Society, Bld. Franklin-Roosevelt, P.O.B. 299, Dakar.

SIERRA LEONE - Sierra Leone Red Cross Society, 6 Liverpool Street, P.O.B. 427, Freetown.

SOMALI REPUBLIC - P.O. Box. 937, Mogadiscio.

SOUTH AFRICA - South African Red Cross, Cor. Kruis \& Market Streets, P.O.B. 8726, Johannesburg.

SPAIN - Spanish Red Cross, Eduardo Dato 16, Madrid, 10.

SUDAN - Sudanese Red Crescent, P.O. Box 235, Khartoum.

SWEDEN - Swedish Red Cross, Artillerigatan 6, 10440, Stockholm 14.

SWITZERLAND - Swiss Red Cross, Taubenstrasse, 8, B.P. 2699, 3001 Berne.

SYRIA - Syrian Red Crescent,13, rue Mahdi Ben Baraka, Damascus.

TANZANIA - Tanzania Red Cross Society, Upanga Road, P.O.B. 1133, Dar es Salaam.

THAILAND - Thai Red Cross Society, King Chulalongkorn Memorial Hospital, Bangkok.

TOGO - Togolese Red Cross Society, Avenue des Alliés 19, P.O. Box 655, Lomé.

TRINIDAD AND TOBAGO - Trinidad and Tobago Red Cross Society, 48 Pembroke Street, P.O. Box 357, Port of Spain.

TUNISIA - Tunisian Red Crescent, 19, rue d'Angleterre, Tunis.

TURKEY - Turkish Red Crescent, Yenisehir, Ankara.

UGANDA - Uganda Red Cross, 57 Roseberry Street, P.O. Box 494, Kampala.

UNITED ARAB REPUBLIC - Red Crescent Society of the United Arab Republic, 34, rue Ramses, Cairo.

UPPER VOLTA - Upper Volta Red Cross, P.O.B. 340, Onagadougou.

URUGUAY - Uruguayan Red Cross, Avenida 8 de Octubre, 2990, Montevideo.

U.S.A. - American National Red Cross, 17th and D Streets, N.W., Washington 6 D.C.

U.S.S.R. - Alliance of Red Cross and Red Crescent Societies, Tcheremushki, J. Tcheremushkinskii proezd 5, Moscow $W$-36.

VENEZUELA - Venezuelan Red Cross, Avenida Andrés Bello No. 4, Apart. 3185, Caracas.

VIET NAM (Democratic Republic) - Red Cross of the Democratic Republic of Viet Nam, 68 , rue Bà-Trièz, Hanoi.

VIET NAM (Republic) - Red Cross of the Republic of Viet Nam, 201, duong HôngThâp-Tu, No. 201, Saigon.

YUGOSLAVIA - Yugoslav Red Cross, Simina ulica broj 19 , Belgrade.

ZAMBIA - Zambia Red Cross, P.O. Box R. W. 1, Ridgeway, Lusaka. 